\title{
Advances in the diagnosis and treatment of gastric neuroendocrine neoplasms
}

\author{
Huangying Tan \\ Department of Integrative Oncology, China-Japan Friendship Hospital, Beijing 100029, China \\ Correspondence to: Huangying Tan. Department of Integrative Oncology, China-Japan Friendship Hospital, Beijing 100029, China. \\ Email: tanhuangying@263.net.
}

\begin{abstract}
Gastric neuroendocrine neoplasms (g-NENs) are a group of heterogeneous tumors arising from the endocrine cells of stomach. Most g-NENs progresses slowly and have a long disease course; however, some other g-NENs grow rapidly, similar to the progression of gastric adenocarcinoma. g-NENs have complex and diverse clinical manifestations and their prognosis and treatment strategies depend highly on clinical subtype, pathological grade, tumour stage, and other factors. Due to their low prevalence, most clinicians have limited knowledge about g-NENs. Missed diagnosis and excessive/inadequate treatment is common in clinical settings. Thus, the diagnosis and treatment of $g$-NENs needs to be further standardized.
\end{abstract}

Keywords: Gastric neuroendocrine neoplasms; g-NENs

Received: 27 October 2016; Accepted: 31 October 2016; Published: 29 November 2016. doi: $10.21037 / \operatorname{tgh} .2016 .11 .03$

View this article at: http://dx.doi.org/10.21037/tgh.2016.11.03

\section{Epidemiology}

According to the United States Surveillance, Epidemiology, and End Results (SEER) database, gastric neuroendocrine neoplasms (g-NENs) accounted for 2.2\% among all NENs for the period from 1950 to 1969 and up to $6 \%$ for the period from 2000 to 2007 (1). The increasing prevalence of g-NENs may be explained by the wider application of gastroscopy, which contributes to the early diagnosis. A prospective study in Austria collected a total of 285 cases of gastrointestinal neuroendocrine tumors from May 2004 to April 2005, yielding an annual incidence of $2.39 / 100,000$, among which g-NENs accounted for $23 \%$ of all gastrointestinal neuroendocrine tumors (2). A prospective study in the Republic of Korea collected a total of 4,951 cases of gastrointestinal neuroendocrine tumors from 2000 to 2009 , among which g-NENs accounted for $14.6 \%$; stomach was the second most commonly affected site (second only to rectum) (3). A research in Argentina indicated that $\mathrm{g}$-NENs accounted for $6.9 \%$ of all gastrointestinal NENs (4). Currently no epidemiological data on $\mathrm{g}$-NENs based on multicenter prospective studies have been available in China.

\section{Pathological diagnostic criteria}

Besides gastroscopic observation with the naked eye, histopathology is essential for the diagnosis of g-NENs. According to the World Health Organization grading criteria of gastroenteropancreatic neuroendocrine tumors (5), NETs can be divided into NET G1, NET G2, NEC G3, and MANEC. In recent years, it has been found that in some patients the tumors may be well-differentiated but the Ki-67 reached G3 (exceeding 20\%, although typically not exceeding $60 \%$ ). Such tumors could not be classified according to the current WHO classification system. In 2013, a Chinese pathologist panel published a consensus document, in which this condition was named as "highly proliferative NETs", with an attempt to distinguish it from NET G3. Table 1 is the World Health Organization grading criteria of gastroenteropancreatic neuroendocrine tumors [2010].

\section{Tumor stage}

Stage is a key prognostic factor for tumors. g-NENs are no exception. The TNM staging system was initially published 
Table 1 The World Health Organization grading criteria of gastroenteropancreatic neuroendocrine tumors [2010]

\begin{tabular}{lcc}
\hline Grade & Mitotic figures (/10 HPF) & Ki-67 index (\% positive) \\
\hline G1 & $<2$ & 2 \\
G2 & $2-20$ & $3-20$ \\
G3 & $>20$ & $>20$ \\
\hline
\end{tabular}

by the European Neuroendocrine Tumor Society (ENETS) in 2006. Later the American Joint Committee on Cancer (AJCC) staging system also describes the TNM of g-NENs. However, definition of $\mathrm{T}$ stage is slightly different between these two staging systems (Table 2). There is no final conclusion which of these two stage systems is better. The specific staging system used in a clinical trial must be clearly stated.

\section{Clinical typing}

In addition to pathological typing and tumor staging, the clinical typing of $\mathrm{g}$-NENs is also very important. Different g-NEN types have dramatically different prognosis and treatment strategies. Classification systems distinguishing three or four types of gastric carcinoid tumor have been proposed: the former divides the welldifferentiated g-NENs into three types $(8,9)$ : type 1 (tumors associated with chronic atrophic gastritis), type 2 (tumors associated with gastrinoma/MEN-1), and type 3 (sporadic lesions). Patients with type 1 or type $2 \mathrm{~g}$-NEN typically have hypergastrinemia; however, type 1 patients have achlorhydria, whereas type 2 patients suffer from excessive gastric acid secretion. Type 3 patients have normal gastrin level and normal gastric acid secretion. The four-type classification, based on the three-type classification, classifies the poorly-differentiated g-NEN and MANEC into type 4 (10-14). We believe the four-type classification is more practical and covers all the g-NENs. The clinicopathological features of each g-NEN type are summarized in Table 3.

\section{Diagnosis}

\section{Gastroscopy and biopsy}

Careful assessment of the tumor and its background mucosa using gastroscopy is particularly important for the typing of g-NENs. Multiple specimens were collected from the tumor, and two or more mucosal specimens were obtained from gastric fundus, gastric body, and gastric antrum (15). For tumors larger than $1 \mathrm{~cm}$, endoscopic ultrasonography is recommended to identify the depth of tumor invasion into the gastric wall and the possible involvement of surrounding lymph nodes.

\section{Pathology}

The pathological diagnosis of g-NENs may follow the criteria proposed in the Concensus on the Pathological Diagnosis of Gastroenteropancreatic Neuroendocrine Tumors in China (2013 edition) (16), with the essential immunohistochemical items including $\mathrm{CgA}$, Syn, and $\mathrm{Ki}-67$. In patients with type $1 \mathrm{~g}-\mathrm{NEN}$, the microscopic findings may include fundal atrophic gastritis, proliferation of neuroendocrine cells, and antral G-cell hyperplasia (17).

\section{Serum gastrin and $\mathrm{Cg} A$}

Serum gastrin is a key indicator for clinical typing and an essential test item for $\mathrm{g}$-NENs. Elevated serum gastrin can be seen in patients with type 1 or type $2 \mathrm{~g}$-NENs; in contrast, the serum gastrin level can be normal in type 3 well-differentiated g-NEN. Serum CgA has certain role in the auxiliary diagnosis and disease monitoring of patients with g-NENs. NSE may increase in poorly-differentiated neuroendocrine carcinoma (type 4).

\section{Twenty four-hour gastric pH monitoring}

Twenty four-hour gastric $\mathrm{pH}$ monitoring may be considered in patient with elevated serum gastrin (18). Patients with type $1 \mathrm{~g}$-NEN have achlorhydria $(\mathrm{pH}>4)$, whereas those with type 2 g-NEN suffer from excessive gastric acid secretion $(\mathrm{pH}<2)$. The gastric secretion is normal in type 3 patients $(\mathrm{pH}<4)$.

\section{Other laboratory tests}

In patients suspicious for type $1 \mathrm{~g}$-NEN, the levels of gastric parietal cell antibody, intrinsic factor antibodies, serum vitamin $B_{12}$, and thyroid function and antibodies may be detected. The gastric parietal cell antibody is positive in about $80 \%$ of type $1 \mathrm{~g}$-NEN patients, whereas some patients may also have serum vitamin $\mathrm{B}_{12}$ deficiency and/ 
Table 2 Definition of T stage and staging system for $g$-NENs in the ENETS and AJCC systems

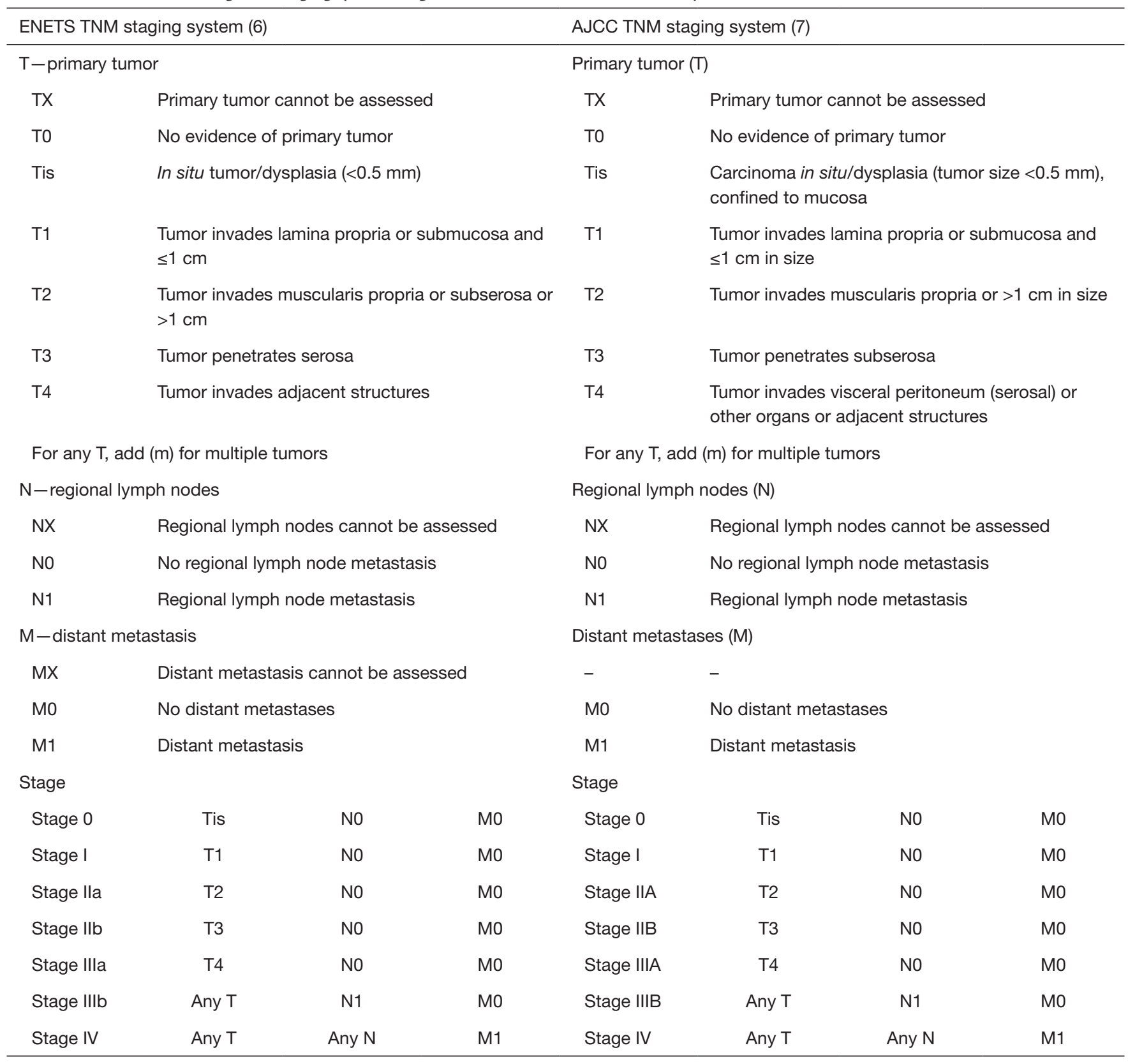

g-NENs, gastric neuroendocrine neoplasms; ENETS, European Neuroendocrine Tumor Society; AJCC, American Joint Committee on Cancer.

or anemia. Some type $1 \mathrm{~g}$-NEN patients may also have autoimmune thyroiditis. In patients suspicious for type 2 g-NEN, the blood pituitary hormones, parathyroid hormone, and calcium (in addition to serum gastrin) should also be measured to rule out the possibility of MEN-1.

\section{Routine imaging}

The type 1 gastric NET has good prognosis and rarely results in metastasis. For the other three types, thoracic/ abdominal contrast-enhanced CT or MRI should be performed to identify any lymph node involvement or 
Table 3 Different types of $\mathrm{g}-\mathrm{NENs}$ and their clinicopathological features (13)

\begin{tabular}{|c|c|c|c|c|}
\hline Main characteristics & Type 1 & Type 2 & Type 3 & Type 4 \\
\hline Associated disease & Type A atrophic gastritis & Gastrinoma/MEN-1 & None & None \\
\hline Site of the tumor & Gastric fundus/body & Gastric fundus/body & Any site & Any site \\
\hline Serum gastrin & Elevated & Elevated & Normal & Mostly normal \\
\hline Number of tumor & Multiple & Multiple & Solitary & Solitary \\
\hline Tumor size & $<1-2 \mathrm{~cm}$ & $<1-2 \mathrm{~cm}$ & Mostly $>2 \mathrm{~cm}$ & $>2 \mathrm{~cm}$ \\
\hline Pathology & Mostly NET G1 & NET G1/G2 & NET G1/G2/G3 & NEC G3 or MANEC \\
\hline Prognosis & Good & Fair & Poor & Extremely poor \\
\hline
\end{tabular}

distant metastasis. In patients suspicious for type $2 \mathrm{~g}$-NEN, corresponding imaging should be performed to locate the g-NENs and rule out the possibility of MEN-1.

\section{Nuclear medicine examinations}

Nuclear medicine examinations include somatostatin receptor scintigraphy (SRS), ${ }^{68} \mathrm{Ga}$-DOTANOC PET/CT, and ${ }^{18} \mathrm{~F}$-FDG PET/CT. Both SRS and ${ }^{68} \mathrm{Ga}$-DOTANOC PET/CT can be used for well-differentiated NENs (the latter is even more sensitive). In contrast, ${ }^{18} \mathrm{~F}$-FDG PET/CT is more feasible for poorly-differentiated NENs (e.g., type 4). The typing algorithm of g-NENs is shown in Figure 1.

\section{Typing-based treatment}

The treatment strategies differ for different g-NEN types.

Type 1 gNET is caused by chronic (autoimmune) atrophic gastritis, achlorhydria, and hypergastrinemia. Gastroscopically it is manifested as mutiple small $(<1-2 \mathrm{~cm})$ polypoid lesions in gastric fundus/body. The prognosis is often good and metastasis is rarely seen, although recurrence is common $(19,20)$. It has been well recognized that conservative treatment (endoscopic excision and follow-up) is better than surgery (21). Procedures of endoscopic resection remain contraversial: most authors suggest that watchful waiting is preferred for gastric lesions sized $1 \mathrm{~cm}$ or smaller and endoscopic resection [endoscopic mucosal resection (EMR) or endoscopic submucosal dissection (ESD)] should only be performed for $\geq 1 \mathrm{~cm}$ lesions. However, others also propose that all the visible small lesions should be removed with biopsy forceps; in particular, lesions sized $\geq 0.5 \mathrm{~cm}$ should be removed by endoscopic resection. No study has compared the effectiveness of proactive endoscopic treatment with that of selective endoscopic resection. In our opinion, lesions sized $\geq 0.5 \mathrm{~cm}$ should be treated by endoscopic resection; for lesions sized $<0.5 \mathrm{~cm}$ (especially multiple lesions), endoscopic treatment is not feasible and endoscopic follow-up or medication is recommended.

For multiple small gastric lesions that are difficult to completely remove under endoscope or lesions that recur repeatedly after endoscopic resection, somatostatin analogs (SSAs) may be used because such drugs can lower serum gastrin, shrink tumors, and reduce relapse (22). Longterm use of SSA is required in patients with type $1 \mathrm{gNET}$ because the disease may recur after the withdrawal of this drug (23). According to our experiences, SSA can be used for 1 year and then stopped for half year, thus achieving the long-term use of this drug at intervals.

Netazepide is a novel gastrin receptor antagonist. It was applied to treat eight patients with multiple type $1 \mathrm{~g}$-NEN in a study (24); after 12 weeks of treatment, the number and size of the tumors decreased and the serum CgA returned normal, although the serum gastrin remained unchanged. This drug was well tolerated. A phase II clinical study of Netazepide further confirmed its efficacy. It may be a promising medical option for type $1 \mathrm{~g}$-NEN patients. 


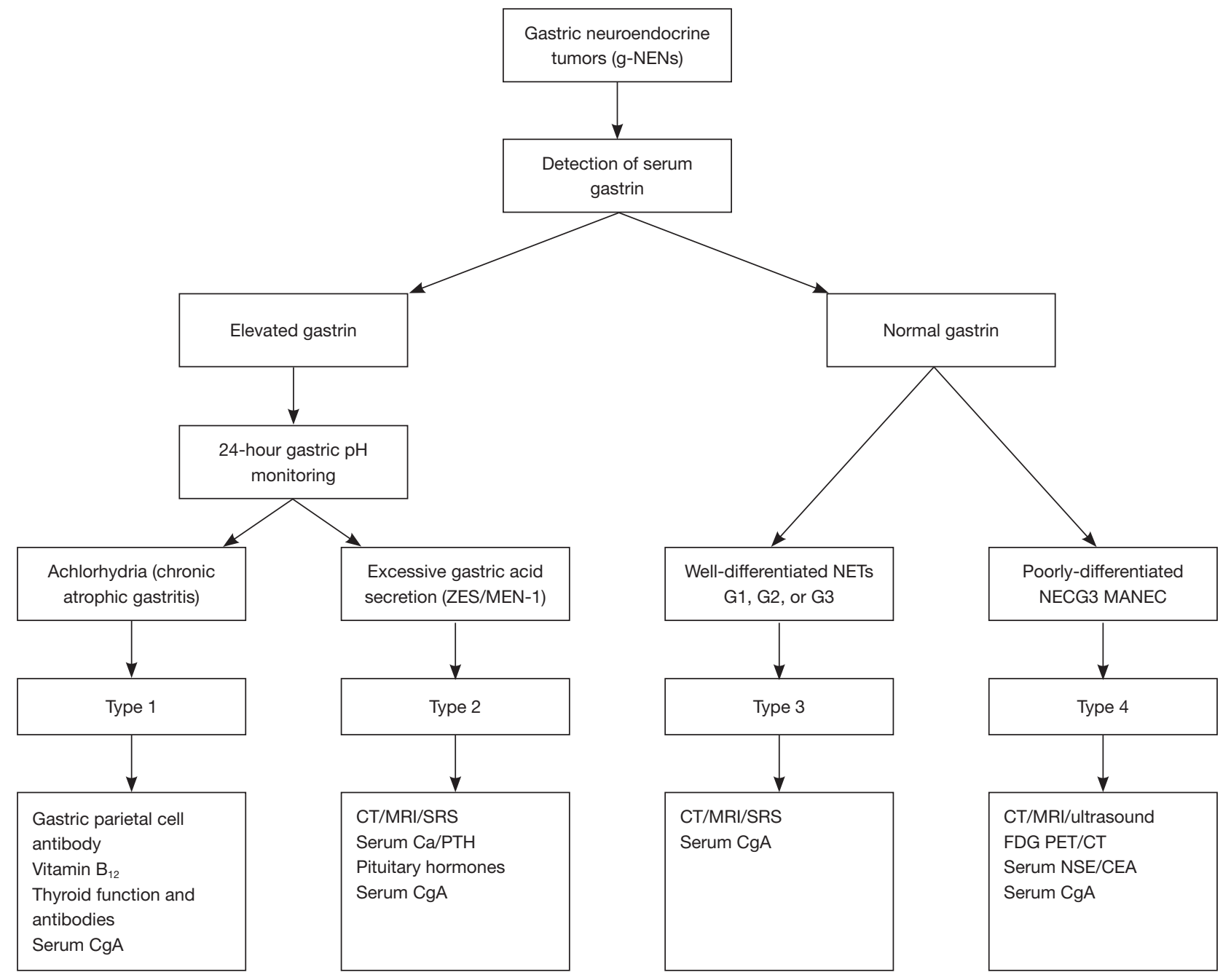

Figure 1 Diagnosis algorithm of gastric NET.

Type $2 \mathrm{~g}$-NEN is mainly treated by surgery, during which both gastrinoma and metastatic lesions were resected. If the multiple metastases cannot be completely removed, SSA therapy may be applied; after the serum gastrin level decreases, the gastric polypoid lesions may also subside. Symptomatic treatment with PPI is often needed in patients with type $2 \mathrm{~g}-\mathrm{NEN}$.

For type 3 g-NEN patients with normal gastrin level, the treatment strategy during the localized phase is similar to that for gastric adenocarcinoma, i.e., partial or total gastrectomy plus lymph node dissection; if the tumor is $\leq 2 \mathrm{~cm}$, endoscopic resection or wedge resection of the stomach may also be feasible. For type $3 \mathrm{~g}$-NEN patients with distant metastasis, SSA is preferred to control the tumor $(25,26)$, with the commonly used drugs including octreotide and lanreotide. These drugs have relatively mild toxicities and can be well tolerated by most patients. Regular ultrasound examinations are needed after longterm use of these drugs, in particular for gallbladder stones. Everolimus may be considered after SSA treatment failure. The RADIANT-4 study has demonstrated that everolimus can prolong the progression-free survival of patients with advanced gastrointestinal and pulmonary NETs (27). Notably, patients with severe accompanying diabetes or lung diseases should not use everolimus. Chemotherapy (preferably temozolomide alone or in combination with capecitabine) should be applied in type $3 \mathrm{~g}$-NEN patients whose pathological diagnosis is "highly proliferative NET 


\section{(NET G3)".}

In patients with poorly-differentiated gastric neuroendocrine carcinoma (type 4), partial or total gastrectomy plus regional lymph node dissection should be performed in patients with localized disease, in whom adjuvant chemotherapy should also be provided after surgery. However, distant metastasis had already occurred at the time of diagnosis in most type 4 patients, in whom the carcinoma is highly malignant and has already become unresectable. Thus, chemotherapy is preferred. EP (etoposide + platinol), the commonly used chemotherapy protocol for small cell lung cancer, has been used as the first-line treatment for type 4 g-NEN, with a response rate of $67 \%$ (28). The secondline treatment options include FOLFOX and FOLRIRI $(29,30)$. Gastric mixed adenoneuroendocrine carcinoma (type 4) is extremely rare and cisplatin-based chemotherapy regimens are recommended (31). The role of SSA in type 4 $\mathrm{g}-\mathrm{NEN}$ patients with positive octreotide scan results remain controversial.

\section{Prognosis and follow-up}

Type 1 g-NEN patients often have good prognosis, in whom metastasis is rarely seen, although the relapse of gastric lesion is common. The median time to relapse was 24 months. Thus, endoscopic follow-up is particularly important and should be performed every 6-12 months. Endoscopic resection is recommended for lesions sized $>0.5 \mathrm{~cm}$ found during the follow-up visits. Meanwhile, serum gastrin and vitamin $B_{12}$ levels should also be monitored. The prognosis is relatively poorer in type 2 patients, in whom the metastasis rate ranges $10-30 \%$ and the mortality rate is $<10 \%$. Gastroscopy should be performed on an annual basis for patients with type 2 g-NEN. In type 3 patients, the prognosis differs depending on the size and metastatic status of the tumor. Patients with tumors in their early stage that can be resected surgically or endoscopically may have good prognosis; however, more than half of the patients with type $3 \mathrm{~g}$-NEN already have metastatic lesions at the time of diagnosis, among whom the tumor-related mortality rate range $25-30 \%$. During the follow-up of type 3 patients with metastasis, the imaging items are same as for gastric adenocarcinoma. Type 4 patients have the poorest prognosis. Up to $80-100 \%$ of type 4 patients already have distant metastasis at the time of diagnosis. The survival is short. Assessment should be performed every 2-3 months during the treatment.

\section{Conclusions}

g-NENs are a group of heterogeneous tumors. Their treatment strategies and prognoses dramatically differ due to their types. The clinical typing of g-NENs should be a priority in clinical practice. Type $1 \mathrm{gNET}$ is a recurrent gastric disease, and its preferred treatment is endoscopic resection and endoscopic follow-up. Not all type 1 g-NEN patients require SSA treatment. For patients with multiple and repeated recurrences, SSA may be considered. However, its high cost and potential toxicities hinders its application. Netazepide, a novel gastrin receptor antagonist, has shown promising efficacy in the treatment of type 1 g-NEN. The future research priorities include the molecular mechanisms of the recurrence of type $1 \mathrm{~g}$-NEN and the R\&D of safe and effective drugs (including TCM medicinals) for controlling the relapse of type $1 \mathrm{~g}$-NEN.

\section{Acknowledgements}

None.

\section{Footnote}

Conflicts of Interest: The author has no conflicts of interest to declare.

\section{References}

1. Yao JC, Hassan M, Phan A, et al. One hundred years after "carcinoid": epidemiology of and prognostic factors for neuroendocrine tumors in 35,825 cases in the United States. J Clin Oncol 2008;26:3063-72.

2. Niederle MB, Hackl M, Kaserer K, et al. Gastroenteropancreatic neuroendocrine tumours: the current incidence and staging based on the $\mathrm{WHO}$ and European Neuroendocrine Tumour Society classification: an analysis based on prospectively collected parameters. Endocr Relat Cancer 2010;17:909-18.

3. Gastrointestinal Pathology Study Group of Korean Society of Pathologists, Cho MY, Kim JM, et al. Current Trends of the Incidence and Pathological Diagnosis of Gastroenteropancreatic Neuroendocrine Tumors (GEPNETs) in Korea 2000-2009: Multicenter Study. Cancer 
Res Treat 2012;44:157-65.

4. O'Connor JM, Marmissolle F, Bestani C, et al. Observational study of patients with gastroenteropancreatic and bronchial neuroendocrine tumors in Argentina: Results from the large database of a multidisciplinary group clinical multicenter study. Mol Clin Oncol 2014;2:673-684.

5. Bosman FT, Carneiro F, Hruban RH, et al. WHO Classification of Tumours of the Digestive System, Fourth Edition. Lyon: International Agency for Research on Cancer (IARC), 2010.

6. Rindi G, Klöppel G, Alhman H, et al. TNM staging of foregut (neuro)endocrine tumors: a consensus proposal including a grading system. Virchows Arch 2006;449:395-401.

7. Compton CC, Byrd DR, Garcia-Aguilar J, et al. editors. AJCC cancer staging atlas. 2nd ed. New York: Springer, 2012.

8. Ruszniewski P, Delle Fave G, et al. Well-differentiated gastric tumors/carcinomas. Neuroendocrinology 2006;84:158-64.

9. Gilligan CJ, Lawton GP, Tang LH, et al. Gastric carcinoid tumors: the biology and therapy of an enigmatic and controversial lesion. Am J Gastroenterol 1995;90:338-52.

10. Rindi G, Bordi C, Rappel S, et al. Gastric carcinoids and neuroendocrine carcinomas: pathogenesis, pathology, and behavior. World J Surg 1996;20:168-72.

11. Scherübl H, Cadiot G, Jensen RT, et al. Neuroendocrine tumors of the stomach (gastric carcinoids) are on the rise: small tumors, small problems? Endoscopy 2010;42:664-71.

12. Park SC, Chun HJ. Clinical Aspects of Gastric and Duodenal Neuroendocrine Neoplasms. Journal of Gastroenterology and Hepatology Research 2012;1:139-46.

13. Tan HY, Lou YN, Luo J, et al. The typing and treatment of gastric neuroendocrine tumors. Chinese Journal of the Frontiers of Medical Science (electronic version) 2014;6:4-8.

14. Liu D, Shen L, Lu M. Diagnosis and treatment for gastric neuroendocrine tumors. Chinese Clinical Oncology 2015;20:549-54.

15. Delle Fave G, Kwekkeboom DJ, Van Cutsem E, et al. ENETS Consensus Guidelines for the management of patients with gastroduodenal neoplasms. Neuroendocrinology 2012;95:74-87.

16. Chinese consensus panel for pathological diagnosis of gastroenteropancreatic tumors. Chinese consensus for pathological diagnosis of gastroenteropancreatic neoplasm. Chinese Journal of Pathology 2013;42:691-4.

17. Chen G, Huang S. Autoimmune metaplastic atrophic gastritis, $\mathrm{G}$ cell hyperplasia and neuroendocrine tumor of stomach. Zhonghua Bing Li Xue Za Zhi 2014;43:34-5.

18. Kunz PL, Reidy-Lagunes D, Anthony LB, et al. Consensus guidelines for the management and treatment of neuroendocrine tumors. Pancreas 2013;42:557-77.

19. Merola E, Sbrozzi-Vanni A, Panzuto F, et al. Type I gastric carcinoids: a prospective study on endoscopic management and recurrence rate. Neuroendocrinology 2012;95:207-13.

20. Grozinsky-Glasberg S, Thomas D, Strosberg JR, et al. Metastatic type 1 gastric carcinoid: a real threat or just a myth? World J Gastroenterol 2013;19:8687-95.

21. Delle Fave G, O'Toole D, Sundin A, et al. ENETS Consensus Guidelines Update for Gastroduodenal Neuroendocrine Neoplasms. Neuroendocrinology 2016;103:119-24.

22. Campana D, Nori F, Pezzilli R, et al. Gastric endocrine tumors type I: treatment with long-acting somatostatin analogs. Endocr Relat Cancer 2008;15:337-42.

23. Jianu CS, Fossmark R, Syversen U, et al. Five-year followup of patients treated for 1 year with octreotide longacting release for enterochromaffin-like cell carcinoids. Scand J Gastroenterol 2011;46:456-63.

24. Moore AR, Boyce M, Steele IA, et al. Netazepide, a gastrin receptor antagonist, normalises tumour biomarkers and causes regression of type 1 gastric neuroendocrine tumours in a nonrandomised trial of patients with chronic atrophic gastritis. PLoS One 2013;8:e76462.

25. Baldelli R, Barnabei A, Rizza L, et al. Somatostatin analogs therapy in gastroenteropancreatic neuroendocrine tumors: current aspects and new perspectives. Front Endocrinol (Lausanne) 2014;5:7.

26. Caplin ME, Pavel M, Ćwikła JB, et al. Lanreotide in metastatic enteropancreatic neuroendocrine tumors. $\mathrm{N}$ Engl J Med 2014;371:224-33.

27. Yao JC, Fazio N, Singh S, et al. Everolimus for the treatment of advanced, non-functional neuroendocrine tumours of the lung or gastrointestinal tract (RADIANT-4): a randomised, placebo-controlled, phase 3 study. Lancet 2016;387:968-77.

28. Strosberg JR, Coppola D, Klimstra DS, et al. The NANETS consensus guidelines for the diagnosis and management of poorly differentiated (high-grade) 
extrapulmonary neuroendocrine carcinomas. Pancreas 2010;39:799-800.

29. Hadoux J, Malka D, Planchard D, et al. Post-first-line FOLFOX chemotherapy for grade 3 neuroendocrine carcinoma. Endocr Relat Cancer 2015;22:289-98.

30. Hentic O, Hammel P, Couvelard A, et al. FOLFIRI regimen: an effective second-line chemotherapy after

doi: $10.21037 / \operatorname{tgh} .2016 .11 .03$

Cite this article as: Tan H. Advances in the diagnosis and treatment of gastric neuroendocrine neoplasms. Transl Gastroenterol Hepatol 2016;1:87. failure of etoposide-platinum combination in patients with neuroendocrine carcinomas grade 3. Endocr Relat Cancer 2012;19:751-7.

31. Pericleous M, Toumpanakis C, Lumgair H, et al. Gastric mixed adenoneuroendocrine carcinoma with a trilineage cell differentiation: case report and review of the literature. Case Rep Oncol 2012;5:313-9. 